\title{
On Sandwich Theorems for Certain Univalent Functions Defined by a New operator
}

\author{
WaggasGalibAtshan ${ }^{1}$ Elaf Ibrahim Badawi ${ }^{2}$ \\ Department of Mathematics,College of Computer Science and Information Technology \\ University of Al-Qadisiyah, Diwaniyah_Iraq \\ waggas.galib@qu.edu.iqwaggashnd@gmail.com¹eilafibraheem911994@gmail.com²
}

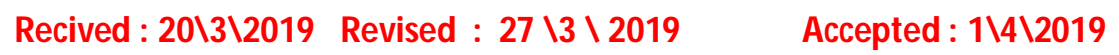

Available online : $30 / 4 / 2019$

\begin{abstract}
:
In this paper, we study some differential subordination and superordination results for certain univalent functions in the open unit disc $\mathrm{U}$ by using a new operator $\mathfrak{f}_{s, a, \mu}^{\lambda}$. Also, we derive some sandwich theorems.
\end{abstract}

Keywords:Analytic function, Differential Subordination, Hadamard Product, Univalentfunction.

Mathematics Subject Classification:30C45 


\section{Introduction}

Denote by $\mathcal{H}=\mathcal{H}(U)$ the class of analytic functions in the open unit disk $U=\{z: z \in \mathbb{C}$ and $z \mid<1\}$. For $n$ a positive integer and $a \in \mathbb{C}$, let $\mathcal{H}[a, n]$ be the subclass of the function $f \in \mathcal{H}$ of the from:

$f(z)=a+a_{n} z^{n}+a_{n+1} z^{n+1}+\cdots \quad(a \in \mathbb{C}, n \in$ $\mathcal{N}=\{1,2,3, \ldots\}) .(1.1)$

Also , Let $T$ be the subclass of $\mathcal{H}$ consisting of functions of the form:

$$
f(z)=z+\sum_{n=2}^{\infty} a_{n} z^{n}(1.2)
$$

If $f \in T$ is given by (1.2) and $g \in T$ given by

$$
g(z)=z+\sum_{k=2}^{\infty} b_{n} z^{n}
$$

The Hadamard product (or the convolution) of $f$ and $g$ is defined by

$$
(f * g)(z)=z+\sum_{k=0}^{\infty} a_{k} b_{k} z^{k}=(g * f)(z) .
$$

If $f$ and $g$ are analytic functions in $U$, We say that $f$ is subordination to $g$.

Let $l, h \in \mathcal{H}$, and $\phi(r, s, t ; z): \mathbb{C}^{3} \times U \rightarrow \mathbb{C}$.

If land $\phi\left(l(z), z l^{\prime}(z), z^{2} l^{\prime \prime}(z) ; z\right)$ are univalent functions in $U$ and if $l$ satisfies the second- order superordination:

$$
h(z) \prec \phi\left(l(z), z l^{\prime}(z), z^{2} l^{\prime \prime}(z) ; z\right),(z \in U)
$$

then $l$ is called a solution of the differential superordination(1.2), (if $f$ subordinate to $g$, then $g$ is superordinate to $f$ ).

An analytic function $q$ is called subordinate of the differential superordination if $q \prec k$ for all $l$ satisfying (1.3). A univalent subordinate $\tilde{q}$ that satisfies $q \prec \tilde{q}$ for all subordinats $q$ of (1.3) is said to be the best subordinat. Recently, Miller and Mocanu [9] obtained sufficient conditions on the functions $(h, k)$ and $\phi$ for which the following implication holds:

$h(z) \prec \phi\left(l(z), z l^{\prime}(z), z^{2} l^{\prime \prime}(z) ; z\right) \Rightarrow q(z) \prec l(z)$,

$(z \in U)$.
Using the results, Bulboacă [5] considered certain classes of first order differential superordinations as well as superordinationpreserving integral operator [6]. Ali et al. [1], have used the results of Bulboacă[5] to obtain sufficient conditions for normalized analytic functions to satisfy:

$$
q_{1}(z) \prec \frac{z f^{\prime}(z)}{f(z)} \prec q_{2}(z),
$$

where $q_{1}$ and $q_{2}$ are given univalent functions in $U$ with $q_{1}(0)=q_{2}(0)=1$. Also, Tuneski [13] obtained a sufficient conditions for starlikeness of $f$ in terms of the quantity

$$
\frac{f^{\prime \prime}(z) f(z)}{\left(f^{\prime}(z)\right)^{2}}
$$

Recently, Shanmugam et al. [11,12] and Goyal et al. [7],Atshan and Hiress[2], Atshan and Kazim[4], Atshan and Jawad [3], Wanas and Majeed [14], also obtained sandwich results for certain classes of analytic functions.

Komatu [8] introduced and investigated a family of integral operator

$\mathfrak{J}_{\mu}^{\lambda}: T \rightarrow T$

that is obtain as follows:

$$
\mathfrak{J}_{\mu}^{\lambda} f(z)=z+\sum_{n=2}^{\infty}\left(\frac{\mu}{\mu+n-1}\right)^{n} a_{n} z^{n}
$$

$\left(z \in U^{*}, \mu>1, \lambda \geq 0\right)$.

The Hurwitiz - Lerch zeta function

$\Phi(z, s, a)=\sum_{k=0}^{\infty} \frac{z^{k}}{(1+a)^{s}}, r \in \mathbb{C} \backslash \mathbb{Z}_{0}^{-}, s \in \mathbb{C} \quad$ when $0<|Z|<1$.

In terms of (Hadamard) product (or convolution) where $G_{s, a(z)}$ is given by

$$
G_{s, a(z)}=(1+a)^{s}\left[\Phi(z, s, a)-a^{-s}\right],(z \in U) .
$$

Definition (2.1.1): Let $f \in T, z \in U^{*}, a \in \mathbb{C} \backslash \mathbb{Z}_{0}^{-}, s \in$ $\mathbb{C}$ and $\lambda>1$,we define a new operator $\mathfrak{f}_{s, a, \mu}^{\lambda} f(z): T \rightarrow T$, where

$$
\mathfrak{f}_{s, a, \mu}^{\lambda} f(z)=G_{s, a}(z) * \mathfrak{I}_{\mu}^{\lambda} f(z)
$$




$$
=z+\sum_{k=2}^{\infty}\left(\frac{1+a}{k+a}\right)^{s}\left(\frac{\mu}{\mu+n-1}\right)^{\lambda} a_{n} z^{n}
$$

We note from (1.6) that

$$
z\left(\mathfrak{f}_{s, a, \mu}^{\lambda+1} f(z)\right)^{\prime}=\mu \mathfrak{f}_{s, a, \mu}^{\lambda} f(z)-(\mu-1) \mathfrak{f}_{s, a, \mu}^{\lambda+1} f(z)
$$

The specific aim of this document is to find sufficientconditions for certain normalized analytic functions $f$ to satisfy:

$$
q_{1}(z) \prec\left(\frac{\rho \mathfrak{f}_{s, a, \mu}^{\lambda+1} f(z)+\xi \mathfrak{f}_{s, a, \mu}^{\lambda} f(z)}{(\rho+\xi) z}\right)^{\frac{1}{\delta}} \prec q_{2}(z),
$$

and

$$
q_{1}(z) \prec\left(\frac{\mathfrak{f}_{s, a, \mu}^{\lambda+1} f(z)}{z}\right)^{\frac{1}{\delta}} \prec q_{2}(z)
$$

where $q_{1}(z)$ and $q_{2}(z)$ are given univalent functions in $U$ with $q_{1}(0)=q_{2}(0)=1$.

\section{Preliminaries}

In order to establish our subordination and superordination results, that require the following lemmas and definitions.

Definition (2.1)[6]: Denote by $Q$ the class of all functions $q$ that are analytic and injective on $\bar{U} \backslash E(q)$, where $\bar{U}=U \cup\{z \in \partial U\}$, and $E(q)=$ $\left\{\zeta \in \partial U: \lim _{z \rightarrow \zeta} f(z)=\infty\right\}$ and are such that $q^{\prime}(\zeta) \neq 0$ for $\zeta \in \partial U \backslash E(q)$. Further, let the subclass of $Q$ for which $q(0)=a$ be denoted by $Q(a)$, $Q(0)=Q_{0}$ and $Q(1)=Q_{1}=\{q \in Q: q(0)=1\}$.

Lemma (2.1)[1]: Let $\mathrm{q}(\mathrm{z})$ be convex univalentfunction in $U$, let $\alpha \in \mathbb{C}, \beta \in \mathbb{C} \backslash\{0\}$ and suppose that

$$
\operatorname{Re}\left\{1+\frac{z q^{\prime \prime}(z)}{q^{\prime}(z)}\right\}>\max \left\{0,-\operatorname{Re}\left(\frac{\alpha}{\beta}\right)\right\} .
$$

If $l(z)$ is analytic in $U$ and

$$
\alpha l(z)+\beta z l^{\prime}(z) \prec \alpha q(z)+\beta z q^{\prime}(z),
$$

then $l(\mathrm{z}) \prec q(z)$ and $q$ is the best dominant.
Lemma (2.2)[3]: Let $q$ be univalent in $U$ and let $\emptyset$ and $\theta$ be analytic in the domain $D$ containing $q(U)$ with $\emptyset(w) \neq 0$, when $w \in q(U)$. Set

$Q(z)=z q^{\prime}(z) \emptyset(q(z)) \operatorname{and} h(z)=\theta(q(z))+Q(z)$,

suppose that

1) $Q$ is starlike univalent in $U$,

$$
\text { 2) } \operatorname{Re}\left(\frac{z h^{\prime}(z)}{Q(z)}\right)>0, z \in U \text {. }
$$

If $l$ is analytic in $U$ with $l(0)=q(0), l(U) \subseteq D$ and

$$
\begin{aligned}
\emptyset(l(z))+z l^{\prime}(z) & \emptyset(l(z)) \\
& \prec \emptyset(q(z))+z q^{\prime}(z) \emptyset(q(z)),
\end{aligned}
$$

then $l(z) \prec q(z)$, and $q$ is the best dominant.

Lemma (2.3)[6]:Let $q(z)$ be convex univalent in the unit disk $U$ and let $\theta$ and $\phi$ be analytic in a domain D containing $q(U)$. Suppose that

$$
\text { 1) } \operatorname{Re}\left\{\frac{\theta^{\prime}(q(z))}{\phi(q(z))}\right\}>0 \text { for } z \in U
$$

2) $Q(z)=z q^{\prime}(z) \phi(q(z))$ is starlike univalent in $z \in U$.

If $l \in \mathcal{H}[q(0), 1] \cap Q$, with $l(U) \subseteq D$, and $\theta(l(z))+$ $z l^{\prime}(z) \phi(l(z))$ is univalent in $U$, and

$\theta(q(z))+z q^{\prime}(z) \phi(q(z)) \prec \theta(l(z))+z l^{\prime}(z) \phi(l(z))$,

then $q(z) \prec l(z)$, and $q$ is the best subordinant.

Lemma (2.4)[6]: Let $q(z)$ be convex univalent in $U$ and $q(0)=1$. Let $\beta \in \mathbb{C}$, that $\operatorname{Re}\{\beta\}>0$. If $l(z) \in$ $\mathcal{H}[q(0), 1] \cap Q$ and $l(z)+\beta z l^{\prime}(z)$ is univalent in $U$, then

$$
q(z)+\beta z q^{\prime}(z) \prec l(z)+\beta z l^{\prime}(z)
$$

which implies that $q(z) \prec l(z)$ and $q(z)$ is the best subordinant .

\section{Subordination Results}

Theorem(3.1): Let $q(z)$ be convex univalent in $U$ with $q(0)=1,0<\delta<1, \eta, \in \mathbb{C} \backslash\{0\}$. Suppose that 
$\operatorname{Re}\left\{1+\frac{z q^{\prime \prime}(z)}{q^{\prime}(z)}\right\}>\max \left\{0,-\operatorname{Re}\left(\frac{1}{\delta \eta}\right)\right\}$.

If $f \in T$ is satisfies the subordination

$$
l(z) \prec q(z)+\delta \eta z q^{\prime}(z),
$$

where

$$
l(z)
$$

$=\left(\frac{\rho \mathfrak{f}_{s, a, \mu}^{\lambda+1} f(z)+\xi \mathfrak{f}_{s, a, \mu}^{\lambda} f(z)}{(\rho+\xi) z}\right)^{\frac{1}{\delta}}(1$

$+\eta\left(\frac{\rho\left[\mu \mathfrak{f}_{s, a, \mu}^{\lambda} f(z)-(\mu-1) \mathfrak{f}_{s, a, \mu}^{\lambda+1} f(z)\right]+\xi\left[\mu \mathfrak{f}_{s, a, \mu}^{\lambda-1} f(z)-(\mu-1) \mathfrak{f}_{s, a, m}^{\lambda} f(z)\right.}{\rho \mathfrak{f}_{s, a, \mu}^{\lambda+1} f(z)+\xi \mathfrak{f}_{s, a, \mu}^{\lambda} f(z)}\right.$

then

$$
\left(\frac{\rho \uparrow_{s, a, \mu}^{\lambda+1} f(z)+\xi \uparrow_{s, a, \mu}^{\lambda} f(z)}{(\rho+\xi) z}\right)^{\frac{1}{\delta}} \prec q(z)
$$

and $q(z)$ is the best dominant.

Proof :consider a function $l(z)$ by

$$
\begin{aligned}
& l(z)=\left(\frac{\rho \uparrow_{s, a, \mu}^{\lambda+1} f(z)+\xi \varlimsup_{s, a, \mu}^{\lambda} f(z)}{(\rho+\xi) z}\right)^{\frac{1}{\delta}} \\
& \times(1
\end{aligned}
$$

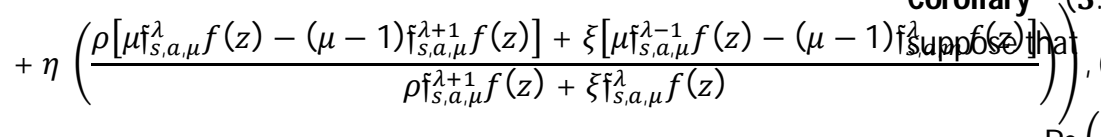

$$
l(z) \prec \frac{1+A z}{1+B z}+\delta \eta \frac{(A-B) z}{(1+B z)^{2}},
$$

wherel ( $z$ ) given by (3.3), then

$$
\left(\frac{\rho f_{s, a, \mu}^{\lambda+1} f(z)+\xi f_{s, a, \mu}^{\lambda} f(z)}{(\rho+\xi) z}\right)^{\frac{1}{\delta}} \prec \frac{1+A z}{1+B z}
$$

and $\frac{1+A z}{1+B z}$ is best dominant.

Taking $A=1$ and $B=-1$ in corollary (3.2), we get following result.

Corollary (3.3): Let $0<\delta<1, \eta \in \mathbb{C} \backslash\{0\}$ and

$$
\begin{aligned}
& l(z)=\left(\frac{\rho \mathfrak{f}_{s, a, \mu}^{\lambda+1} f(z)+\xi \mathfrak{f}_{s, a, \mu}^{\lambda} f(z)}{(\rho+\xi) z}\right)^{\frac{1}{\delta}} \\
& \times(1
\end{aligned}
$$$$
\operatorname{Re}\left(\frac{1+z}{1-z}\right)>\max \{0,-\operatorname{Re}(\delta \eta)\} \text {. }
$$

If $f \in T$ is satisfy the following subordination

$$
l(z) \prec \frac{1+z}{1-z}+\delta \eta \frac{2 z}{(1-z)^{2}},
$$

wherel $(z)$ given by (3.3), then

$$
\left(\frac{\rho \mathfrak{f}_{s, a, \mu}^{\lambda+1} f(z)+\xi \mathfrak{f}_{s, a, \mu}^{\lambda} f(z)}{(\rho+\xi) z}\right)^{\frac{1}{\delta}} \prec \frac{1+z}{1-z}
$$

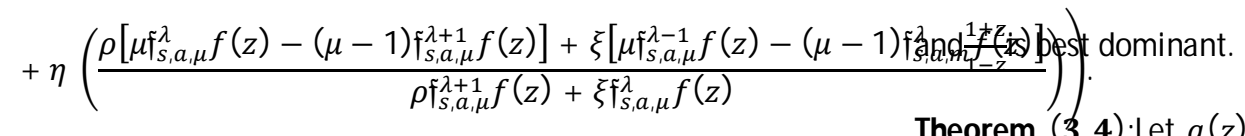

Theorem (3.4): Let $q(z)$ be convex univalent in unit disk $U$ with $q(0)=1$, let $0<\delta<1, \eta \in$ 
$\mathbb{C} \backslash\{0\}, u, v, \varepsilon, \alpha \in \mathbb{C}, f \in T$ and suppose that $f$ and $q$ satisfy the following conditions

$\operatorname{Re}\left\{\frac{v}{\eta} q(z)+\frac{2 \varepsilon}{\eta}[q(z)]^{2}+1+\frac{3 \alpha}{\eta}[q(z)]^{3}+z \frac{q^{\prime \prime}(z)}{q^{\prime}(z)}-\right.$

$\left.z \frac{q^{\prime}(z)}{q(z)}\right\}>0$

and

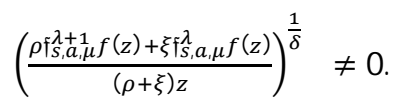

If

$r(z) \prec u+v q(z)+\varepsilon[q(z)]^{2}+\alpha[q(z)]^{3}+$

$\eta \frac{z q^{\prime}(z)}{q(z)}$

where

$$
\begin{gathered}
r(z)=u+v\left(\frac{\rho f_{s, a, \mu}^{\lambda+1} f(z)+\xi f_{s, a, \mu}^{\lambda} f(z)}{(\rho+\xi) z}\right)^{\frac{1}{\delta}}+ \\
\varepsilon\left(\frac{\rho f_{s, a, \mu}^{\lambda+1} f(z)+\xi f_{s, a, \mu}^{\lambda} f(z)}{(\rho+\xi) z}\right)^{\frac{1}{2 \delta}}+\alpha\left(\frac{\rho f_{s, a, \mu}^{\lambda+1} f(z)+\xi f_{s, a, \mu}^{\lambda} f(z)}{(\rho+\xi) z}\right)^{\frac{1}{3 \delta}}+ \\
\frac{1}{\eta \delta}\left[\frac{\rho\left(f_{s, a, \mu}^{\lambda+1} f(z)\right)^{\prime}+\xi\left(f_{s, a, \mu}^{\lambda} f(z)\right)^{\prime}}{\rho f_{s, a, \mu}^{\lambda+1} f(z)+\xi \uparrow_{s, a, \mu}^{\lambda} f(z)}-1\right]
\end{gathered}
$$

then

$\left(\frac{\rho \uparrow_{s, a, \mu}^{\lambda+1} f(z)+\xi \uparrow_{s, a, \mu}^{\lambda} f(z)}{(\rho+\xi) z}\right)^{\frac{1}{\delta}} \prec q(z)$, and $q(z)$ is best dominant.

Proof :consider a function $l(z)$ by

$$
l(z)=\left(\frac{\rho \uparrow_{s, a, \mu}^{\lambda+1} f(z)+\xi \uparrow_{s, a, \mu}^{\lambda} f(z)}{(\rho+\xi) z}\right)^{\frac{1}{\delta}}
$$

Then the function $p$ is analytic in $U$ and $l(0)=1$, differentiating (3.10) logarithmically with respect to $z$, we get

$\frac{z l^{\prime}(z)}{l(z)}=\frac{1}{\delta}\left[\frac{\rho\left(\uparrow_{s, a, \mu}^{\lambda+1} f(z)\right)^{\prime}+\xi\left(f_{s, a, \mu}^{\lambda} f(z)\right)^{\prime}}{\rho \uparrow_{s, a, \mu}^{\lambda+1} f(z)+\xi \uparrow_{s, a, \mu}^{\lambda} f(z)}-1\right] .(3$

By setting $\theta(w)=u+v w+\varepsilon w^{2}+\alpha w^{3} \quad$ and $\phi(w)=\frac{\eta}{w}$, it can be easily observed that $\theta(w)$ is analytic in $\mathbb{C}, \phi(w)$ is analytic in $\mathbb{C} \backslash\{0\}$ and that $\phi(w) \neq 0, w \in \mathbb{C} \backslash\{0\}$. Also, we get

$$
Q(z)=z q^{\prime}(z) \phi(q(z))=\eta \frac{z q^{\prime}(z)}{q(z)}
$$

and

$$
\begin{gathered}
h(z)=\theta(q(z))+Q(z) \\
=u+v q(z)+\varepsilon[q(z)]^{2}+\alpha[q(z)]^{3}+\eta \frac{z q^{\prime}(z)}{q(z)},
\end{gathered}
$$

It is observe that $Q(z)$ is starlike univalent in $U$, we have

$$
\begin{aligned}
\operatorname{Re}\left(\frac{z h^{\prime}(z)}{Q(z)}\right)= & \operatorname{Re}\left(\frac{v}{\eta} q(z)+\frac{2 \varepsilon}{\eta}[q(z)]^{2}+\frac{3 \alpha}{\eta}[q(z)]^{3}\right. \\
& \left.+1+\frac{z q^{\prime \prime}(z)}{q^{\prime}(z)}-\frac{z q^{\prime}(z)}{q(z)}\right)>0 .
\end{aligned}
$$

By Making use of (2.2), we obtain

$$
\begin{gathered}
v l(z)+\varepsilon[l(z)]^{2}+\alpha[l(z)]^{3} \prec \\
v q(z)+\varepsilon[q(z)]^{2}+\alpha[q(z)]^{3}+\eta \frac{z q^{\prime}(z)}{q(z)}
\end{gathered}
$$

and by using Lemma (2.2), we deduce that subordination (3.8) implies that

$l(z) \prec q(z)$ and the function $q(z)$ is the best dominant.

Taking the function $(z)=\frac{1+A z}{1+B z}(-1 \leq B<A \leq$ 1), in Theorem (3.4) for every $\eta \in \mathbb{C} \backslash\{0\}$ the condition(3.6)becomes

$$
\begin{aligned}
& \operatorname{Re}\left(\frac{v}{\eta} \frac{1+A z}{1+B z}+\frac{2 \varepsilon}{\eta}\left(\frac{1+A z}{1+B z}\right)^{2}+\frac{2 \alpha}{\eta}\left(\frac{1+A z}{1-B z}\right)^{3}+1+\right. \\
& \left.\frac{(A-B) z}{(1+B z)(1+A z)}-\frac{2 B z}{1+B z}\right)>0,(3.12)
\end{aligned}
$$

hence, we have the following corollary.

Corollary(3.5): Let $(-1 \leq B<A \leq 1), 0<\delta<1, \eta \in$ $\mathbb{C} \backslash\{0\}, u, v, \varepsilon, \alpha \in \mathbb{C}$.

Assume that (3.12) holds.

If $f \in T$ and

$$
\begin{gathered}
r(z) \prec u+v \frac{1+A z}{1+B z}+\varepsilon\left(\frac{1+A z}{1+B z}\right)^{2}+\alpha\left(\frac{1+A z}{1+B z}\right)^{3} \\
+\eta \frac{(A-B) z}{(1+B z)(1+A z)},
\end{gathered}
$$


where $r(z)$ is defined in (3.9), then

$\left(\frac{\mathfrak{f}_{s, a, \mu}^{\lambda+1} f(z)}{z}\right)^{\frac{1}{\delta}} \prec \frac{1+A z}{1+B z}$, and $\frac{1+A z}{1+B z}$ is best dominant.

Taking the function $q(z)=\left(\frac{1+z}{1-z}\right)^{\rho}$

$(0<\rho \leq 1)$, in Theorem(3.4), the condition

\section{(2.12)becomes}

$\operatorname{Re}\left\{\frac{v}{\eta}\left(\frac{1+z}{1-z}\right)^{\rho}+\frac{2 \varepsilon}{\eta}\left(\frac{1+z}{1-z}\right)^{2 \rho}+\frac{\alpha}{\eta}\left(\frac{1+z}{1-z}\right)^{3 \rho}+\frac{2 z^{2}}{1-z^{2}}\right\}>$ $0(\eta \in \mathbb{C} \backslash\{0\})$,

hence, we have the following corollary.

Corollary (3.6): Let $\quad 0<\rho \leq 1,0<\delta<1, \eta \in$ $\mathbb{C} \backslash\{0\}, u, v, \varepsilon, \alpha \in \mathbb{C}$. Assume that (3.13) holds. If $f \in$ $T$ and

$$
\begin{gathered}
r(z) \prec u+v\left(\frac{1+z}{1-z}\right)^{\rho}+\varepsilon\left(\frac{1+z}{1-z}\right)^{2 \rho}+\alpha\left(\frac{1+z}{1-z}\right)^{3 \rho} \\
+\eta \frac{2 \rho z}{1-z^{2}}, r(z)=
\end{gathered}
$$

where $r(z)$ is defined in (3.9), then

$\left(\frac{\mathfrak{f}_{s, a, \mu}^{\lambda+1} f(z)}{z}\right)^{\frac{1}{\delta}} \prec\left(\frac{1+z}{1-z}\right)^{\rho}$, and $\left(\frac{1+z}{1-z}\right)^{\rho}$ is best dominant.

\section{Superordination Results}

Theorem (4.1): Let $q(z)$ be convex univalent in $U$ with $q(z)=1,0<\delta<1, \eta \in \mathbb{C}$ with $\operatorname{Re}(\eta)>0, \quad$ if $f \in T$, such that

$$
\left(\frac{\rho \mathfrak{f}_{s, a, \mu}^{\lambda+1} f(z)+\xi \mathfrak{f}_{s, a, \mu}^{\lambda} f(z)}{(\rho+\xi) z}\right)^{\frac{1}{\delta}} \neq 0
$$

and suppose that $f$ satisfies the condition:

$$
\begin{gathered}
\left(\frac{\rho \mathfrak{f}_{s, a, \mu}^{\lambda+1} f(z)+\xi \mathfrak{f}_{s, a, \mu}^{\lambda} f(z)}{(\rho+\xi) Z}\right)^{\frac{1}{\delta}} \\
\in \mathcal{H}[q(0), 1] \cap Q .
\end{gathered}
$$

If the function $l(z)$ given by (3.3) is univalent and the following superordination condition:

$q(z)+\delta \eta z q^{\prime}(z) \prec l(z),(4.2)$

holds, then

$$
q(z) \prec\left(\frac{\rho \uparrow_{s, a, \mu}^{\lambda+1} f(z)+\xi \digamma_{s, a, \mu}^{\lambda} f(z)}{(\rho+\xi) z}\right)^{\frac{1}{\delta}}(4
$$

and $q(z)$ is the best subordinant.

Proof : Consider the analytic function $l(z)$ by

$$
l(z)=\left(\frac{\rho \uparrow_{s, a, \mu}^{\lambda+1} f(z)+\xi \uparrow_{s, a, \mu}^{\lambda} f(z)}{(\rho+\xi) z}\right)^{\frac{1}{\delta}} .
$$

Differentiate Euquation(4.4) with the respect to $Z$ logarithmically, we get

$\frac{z l^{\prime}(z)}{l(z)}=\frac{1}{\delta}\left(\frac{\rho z\left(\mathfrak{f}_{s, a, \mu}^{\lambda+1} f(z)\right)^{\prime}+\xi z\left(\mathfrak{f}_{s, a, \mu}^{\lambda} f(z)\right)^{\prime}}{\rho \mathfrak{f}_{s, a, \mu}^{\lambda+1} f(z)+\xi \mathfrak{f}_{s, a, \mu}^{\lambda}(z)}\right)(4.5$

A simple computation and using (1.6), from (4.5), we get

$l(z)$

$=\left(\frac{\rho \mathfrak{f}_{a, \mu}^{s, \lambda+1} f(z)+\xi \mathfrak{f}_{a, \mu}^{s, \lambda} f(z)}{(\rho+\xi) z}\right)^{\frac{1}{\delta}}$

$\times(1$

$+\eta\left(\frac{\rho\left[\mu \mathfrak{f}_{a, \mu}^{s, \lambda+1} f(z)-(\mu-1) \mathfrak{f}_{a, \mu}^{s, \lambda} f(z)\right]+\xi\left[\mu \mathfrak{f}_{a, \mu}^{s, \lambda} f(z)-(\mu-1) \mathfrak{f}_{a, m}^{s, \lambda-}\right.}{\rho \mathfrak{f}_{a, \mu}^{s, \lambda+1} f(z)+\xi \mathfrak{f}_{a, \mu}^{s, \lambda} f(z)}\right.$

$=l(z)+\delta \eta z l^{\prime}(z)$

now, by using Lemma (2.4), we get the desired result.

Taking $q(z)=\frac{1+A z}{1+B z}(-1 \leq B<A \leq 1)$, in Theorem (4.1), we get thefollowing corollary.

Corollary (4.2): Let $\operatorname{Re}\{\eta\}>0,0<\delta<1 \quad$ and $-1 \leq B<A \leq 1$, such that

$$
\left(\frac{\rho \mathfrak{f}_{s, a, \mu}^{\lambda+1} f(z)+\xi \mathfrak{f}_{s, a, \mu}^{\lambda} f(z)}{(\rho+\xi) z}\right)^{\frac{1}{\delta}} \in \mathcal{H}[q(0), 1] \cap Q
$$

If the function $l(z)$ given by (3.3) is univalent in $U$ and $f \in T$ satisfies the following superordination condition:

$\frac{1+A z}{1+B z}+\delta \eta \frac{(A-B) z}{(1+B z)^{2}} \prec l(z)$, then 


$$
\frac{1+A z}{1+B z} \prec\left(\frac{\rho \mathfrak{f}_{s, a, \mu}^{\lambda+1} f(z)+\xi \mathfrak{f}_{s, a, \mu}^{\lambda} f(z)}{(\rho+\xi) z}\right)^{\frac{1}{\delta}},
$$

and the function $\frac{1+A z}{1+B z}$ is the bestsubordinant.

Theorem (4.3): Let $q(z)$ be convex univalent in unit $\operatorname{disk} U$, let $\eta \in \mathbb{C} \backslash\{0\}, 0<\delta<1, u, v, \varepsilon \in \mathbb{C}, q(z) \neq 0$, and $f \in T$. Suppose that

$$
\operatorname{Re}\left\{(v+2 \varepsilon q(z)+3 \alpha q(z)) \frac{q(z) q^{\prime}(z)}{\eta}\right\}>0,
$$

Let $f(z) \in T$ and suppose that satisfies the next condition:

$$
\left(\frac{\rho \rho_{s, a, \mu}^{\lambda+1} f(z)+\xi \digamma_{s, a, \mu}^{\lambda} f(z)}{(\rho+\xi) z}\right)^{\frac{1}{\delta}} \in \mathcal{H}[q(0), 1] \cap Q,(4.6)
$$

and

$$
\frac{\rho \mathfrak{f}_{s, a, \mu}^{\lambda+1} f(z)+\xi \mathfrak{f}_{s, a, \mu}^{\lambda} f(z)}{(\rho+\xi) z} \neq 0 .
$$

If the function $r(z)$ is given by (3.9) is univalent in $U$, and

$\mathrm{u}+v q(z)+\varepsilon[q(z)]^{2}+\alpha[q(z)]^{3}+\eta \frac{z q^{\prime}(z)}{q(z)} \prec r(z)$,

implies

$$
q(z) \prec\left(\frac{\rho \mathfrak{f}_{s, a, \mu}^{\lambda+1} f(z)+\xi \mathfrak{f}_{s, a, \mu}^{\lambda} f(z)}{(\rho+\xi) z}\right)^{\frac{1}{\delta}}
$$

and $q(z)$ is the best subordinant.

Proof : Let the function $A(z)$ defined on $U$ by (3.14). Then a computation show that

$\frac{z l^{\prime}(z)}{l(z)}=\frac{1}{\delta}\left[\frac{\rho\left(f_{s, a, \mu}^{\lambda+1} f(z)\right)^{\prime}+\xi\left(f_{s, a, \mu}^{\lambda} f(z)\right)^{\prime}}{\rho f_{s, a, \mu}^{\lambda+1} f(z)+\xi f_{s, a, \mu}^{2} f(z)}-1\right](4.8)$

By setting $\theta(w)=u+v w+\varepsilon w^{2}+\alpha w^{3} \quad$ and $\phi(w)=\frac{\eta}{w}$, it can be easily observed that $\theta(w)$ is analytic in $\mathbb{C}, \phi(w)$ is analytic in $\mathbb{C}\{0\}$ and that $\phi(w) \neq 0(w \in \mathbb{C} \backslash\{0\})$. Also, we get $Q(z)=$ $z q^{\prime}(z) \phi(q(z))=\eta \frac{z q^{\prime}(z)}{q(z)}$, it observed that $Q(z)$ is starlike univalent in $U$.
Since $q(z)$ is convex, it follows that

$$
\begin{aligned}
\operatorname{Re}\left\{\frac{\theta^{\prime}(q(z))}{\phi(q(z))}\right\}= & \operatorname{Re}\left\{\frac { q ( z ) } { \eta } \left(2 \varepsilon q(z)+3 \alpha[q(z)]^{2}\right.\right. \\
& +v)\} q^{\prime}(z)>0
\end{aligned}
$$

By making use of (4.8) the hypothesis (4.7) can be equivalently written as

$$
\begin{aligned}
\theta\left(q(z)+z q^{\prime}(z) \phi(q(z))\right) & \\
& =\theta\left(l(z)+z l^{\prime}(z) \phi(A(z))\right),
\end{aligned}
$$

thus, by applying Lemma (2.3), the proof is completed.

\section{Sandwich Results}

Combining Theorem (3.1) with Theorem (4.1), we obtain the following sandwich Theorem :

Theorem (5. 1): Let $q_{1}$ and $q_{2}$ be convex univalent in $U$ with $q_{1}(0)=q_{2}(0)=1$ and $q_{2}$ satisfies (3.1). Suppose that $\operatorname{Re}\{\eta\}>0,0<\delta<1, \eta \in \mathbb{C} \backslash\{0\}$.

If $f \in T$, such that

$$
\left(\frac{\rho \uparrow_{s, a, \mu}^{\lambda+1} f(z)+\xi \mathfrak{f}_{s, a, \mu}^{\lambda} f(z)}{(\rho+\xi) z}\right)^{\frac{1}{\delta}} \in \mathcal{H}[q(0), 1] \cap Q,
$$

and the function $l(z)$ defined by (3.3) is univalent and satisfies

$$
\begin{aligned}
q_{1}(z)+\delta \eta z q_{1}^{\prime}(z) & \prec l(z) \\
& \prec q_{2}(z)+\delta \eta z q_{2}^{\prime}(z),(5.1)
\end{aligned}
$$

implies that

$$
q_{1}(z) \prec\left(\frac{\rho \overbrace{s, a, \mu}^{\lambda+1} f(z)+\xi \varlimsup_{s, a, \mu}^{\lambda} f(z)}{(\rho+\xi) z}\right)^{\frac{1}{\delta}} \prec q_{2}(z),
$$

where $q_{1}$ and $q_{2}$ are, respectively , the best subordinant and the best dominant of (5.1).

Combining Theorem (3.4) with Theorem (4.3), we obtain the following sandwich Theorem.

Theorem (5.2):Let $q_{i}$ be two convex univalent functions in $U$, such that $q_{i}(0)=1, q_{i}(0) \neq$ $0(i=1,2)$. Suppose that $q_{1}$ and $q_{2}$ satisfies (4.8) and (3.8), respectively. 
If $f \in T$ and suppose that $f$ satisfies the next conditions:

$$
\begin{gathered}
\left(\frac{\rho \mathfrak{f}_{s, a, \mu}^{\lambda+1} f(z)+\xi \mathfrak{f}_{s, a, \mu}^{\lambda} f(z)}{(\rho+\xi) z}\right)^{\frac{1}{\delta}} \neq 0 \\
\text { and } \\
\left(\frac{\rho \mathfrak{f}_{s, a, \mu}^{\lambda+1} f(z)+\xi \mathfrak{f}_{s, a, \mu}^{\lambda} f(z)}{(\rho+\xi) z}\right)^{\frac{1}{\delta}} \in \mathcal{H}[q(1), 1] \cap Q,
\end{gathered}
$$

and $r(z)$ is univalent in $U$, then

$$
\begin{aligned}
& \mathrm{u}+v q_{1}(z)+\varepsilon\left[q_{1}(z)\right]^{2}+\alpha\left[q_{1}(z)\right]^{3}+\eta \frac{z q_{1}^{\prime}(z)}{q_{1}(z)} \prec \\
& r(z) \prec \mathrm{u}+v q_{2}(z)+\alpha\left[q_{2}(z)\right]^{3}+\eta \frac{z q_{2}^{\prime}(z)}{q_{2}(z)}(5.2)
\end{aligned}
$$

implies

$$
q_{2}(z) \prec\left(\frac{\rho \mathfrak{f}_{s, a, \mu}^{\lambda+1} f(z)+\xi \mathfrak{f}_{s, a, \mu}^{\lambda} f(z)}{(\rho+\xi) z}\right)^{\frac{1}{\delta}} \prec q_{2}(z),
$$

and $q_{1}$ and $q_{2}$ are the best subordinant and the best dominant respectively and $r(z)$ is given by (3.9).

\section{References:}

[1]R. M. Ali, V. Ravichandran, M. H. Khan and K. G. Subramaniam, Differential sandwich theorems for certain analytic functions, Far East J. Math. Sci., 15(1) (2004), 87-94.

[2] W. G. Atshan, R. A. Hiress, On Differential Sandwich theorems of Meromorphic univalent functions, Journal of Al-Qadisiyah for Computer Science and Mathematics, Vol (10),no(3),(2018).

[3]W. G. Atshan, S. A. Jawad, On Differential Sandwich Results for analytic functions, Journal of Al-Qadisiyah for Computer Science and Mathematics, Vol.(11),No.(1),(2019),96-101.

[4] W. G. Atshan, S. K. Kazim,On Differential sandwich Theorems of Mulivalent Functions Defined a Linear Operator, Journal of Al-Qadisiyah for Computer Science and Mathematics, Vol.(10),No.(1),(2019), 117-123.
[5] T. Bulboacă, Classes of first-order differential superordinations, Demonstration Math., 35(2) (2002), 287-292.

[6] T. Bulboacă, Differential Subordinations and Superordinations, Recent Results, House of Scientific Book Publ., Cluj-Napoca, (2005 ).

[7] S. P. Goyal, P. Goswami and H. Silverman, Subordination and superordination results for a class of analytic multivalent functions, Int. J. Math. Math. Sci. (2008), Article ID 561638,1-12.

[8] Y. Komatu, On analytic prolongation of a family of integral operators, Mathematica (cluj), 32(55)(1990), 141-145.

[9] S. S. Miller and P. T. Mocanu, Differential subordinations: Theory and Applications, Series on Monographs and Text Books in Pure and Applied Mathematics, 225, Marcel Dekker, New York and Basel, (2000).

[10]S. S. Miller and P. T. Mocanu, Subordinations of differential inequalities superordinations, Complex Variables,48(10), $815-826,(2003)$.

[11]T. N. Shanmugam, V. Ravichandran and S. Sivasubramanian, Differential sandwich theorems for subclasses of analytic functions, Aust. J. Math. Anal. Appl., 3 (2006), Article 8,1-11

[12]T. N. Shanmugam, S. Shivasubramanian and $H$. Silverman, On sandwich theorems for some classes of analytic functions, Int. J. Math. Math. Sci. (2006), Article ID 29684, 1 - 13.

[13]N. Tuneski, On certain sufficient conditions for starlikeness, Internat. J. Math. Sci. 23(8), (2000), 521-527.

[14]A. K. Wanas and A. H. Majeed ,Differential sandwich theorems for multi valentanalytic functions defined by convolution structure with generalized hypergeometric function, AnaleeUniv.Oradea Fasc. Math., XXV(2)(2018),37-52. 
حول مبرهنات الساندوج للاو ال الاحادية التكافؤ الاكيدة و المعرفة بواسطة مؤثر جليد

$$
\text { وقاص غالب عطشان } 1 \text { إيلاف إبراهيم بديوي2 }
$$

قسم الرياضيات ،كلية علوم الحاسوب وتكنولوجيا المعلومات ،جامعة القادسية ، الديوانيةـ العراق

المستخلص:

في هذا البحث درسنا بعض نتائج التبعية التفاضلية العليا للاو ال احادية التكافؤ الاكيدففي قرص الوحدة المفتوح باستخدام مؤثر جديد f مبر هنات الساندوج. 